\title{
Cooper triples in attractive three-component fermions: Implication for hadron-quark crossover
}

\author{
Hiroyuki Tajima $\odot,{ }^{1}$ Shoichiro Tsutsui, ${ }^{2}$ Takahiro M. Doi, ${ }^{3,4}$ and Kei Iida ${ }^{1}$ \\ ${ }^{1}$ Department of Mathematics and Physics, Kochi University, Kochi 780-8520, Japan \\ ${ }^{2}$ Quantum Hadron Physics Laboratory, RIKEN Nishina Center, Wako, Saitama 351-0198, Japan \\ ${ }^{3}$ Research Center for Nuclear Physics (RCNP), Osaka University, 567-0047, Japan \\ ${ }^{4}$ RIKEN iTHEMS, Wako, Saitama 351-0198, Japan
}

(Received 7 December 2020; revised 1 March 2021; accepted 24 January 2022; published 18 February 2022)

\begin{abstract}
We investigate many-body properties of equally populated three-component fermions with attractive threebody contact interaction in one dimension. A diagrammatic approach suggests the possible occurrence of Cooper triples at low temperature, which are three-body counterparts of Cooper pairs with a two-body attraction. We develop a minimal framework that bridges the crossover from tightly bound trimers to Cooper triples with increasing chemical potential and show how the formation of Cooper triples occurs in the grand-canonical phase diagram. Moreover, we argue that this nontrivial crossover is similar to the hadron-quark crossover proposed in dense matter. A coexistence of medium-induced triples and the underlying Fermi sea at positive chemical potential is analogous to quarkyonic matter consisting of baryonic excitations and the underlying quark Fermi sea. The comparison with the existing quantum Monte Carlo results implies that the emergence of these kinds of three-body states can be a microscopic origin of the peak of the sound velocity along the crossover.
\end{abstract}

DOI: 10.1103/PhysRevResearch.4.L012021

Introduction-The Cooper problem, where two-component fermions with a two-body attraction undergo an instability toward superconductivity, brought about a significant breakthrough in condensed matter and particle physics [1]. On the other hand, three-body and higher-body interactions occurring among particles with internal degrees of freedom play a significant role in cold atomic and nuclear physics [2-6].

In ultracold atoms, the importance of the residual threebody interaction in a one-dimensional (1D) system $[7,8]$ and resulting trimer formation [9] have been pointed out. Moreover, not only the realization of non-negligible multibody interactions [10-13], but also various related phenomena have been proposed [14-19]. Recently, the conditions for attractive and repulsive three-body interactions [20] and the Bose-Fermi duality including three-body forces have been discussed [21-23].

Other interesting aspects of the three-body interaction are the emergences of a quantum scale anomaly and an asymptotic freedom in nonrelativistic 1D three-component fermions [24]. In fact, such a system possesses scale invariance classically [25,26], while this scale invariance is broken by the presence of three-body quantum bound states. This anomaly is associated with the asymptotic freedom according to which the running coupling constant becomes progressively weaker in a high-energy regime as in quantum chromodynamics (QCD) [27]. The same anomaly also

Published by the American Physical Society under the terms of the Creative Commons Attribution 4.0 International license. Further distribution of this work must maintain attribution to the author(s) and the published article's title, journal citation, and DOI. emerges in two-dimensional (2D) fermions with two-body attraction [28-35]. At low density, the molecular bosonic condensate has been observed in the 2D system [36], while a gas of Fermi degenerate trimers is expected to be realized in the 1D system [24,37-40]. Even at high density, the 2D system undergoes a Cooper-pair condensation. In the 1D system, however, the three-body counterparts of Cooper pairs remain to be explored. A candidate is a Cooper triple (see Fig. 1) predicted in three-dimensional (3D) three-component Fermi gases with two-body attraction $[41,42]$. It is important to see the stability of such an exotic state, given that the medium effect on Efimov trimer states is non-negligible [41-47].

In dense QCD, moreover, Cooper triples might be relevant to the hadron-quark continuity $[48,49]$ because quarks are three-component fermions in color space. So far, various scenarios have been discussed in connection with recent astrophysical observations. One of the intriguing state is quarkyonic matter [50], which has been proposed to describe the intermediate-density regime as a state in which quark and baryonic degrees of freedom coexist in the course of the hadron-quark crossover [48,49] where typical energyscale separations occur among the Debye screening mass $m_{\mathrm{D}}$, QCD energy scale $\Lambda_{\mathrm{QCD}}$, and quark chemical potential $\mu_{q}$ as $m_{\mathrm{D}} \ll \Lambda_{\mathrm{QCD}} \ll \mu_{q}[50,51]$. Another interesting picture called percolation has also been proposed, where quark deconfinement starts with formation of a percolation network $[5,6]$. While such states have been investigated phenomenologically and the resulting equation of state is consistent with recent astrophysical observations of neutron stars [6,52-54], a microscopic mechanism of these many-body phenomena is not obvious even at a qualitative level. Thus, it will be interesting if there is a connection between baryonic excitations in dense QCD and possible color Cooper triples. 


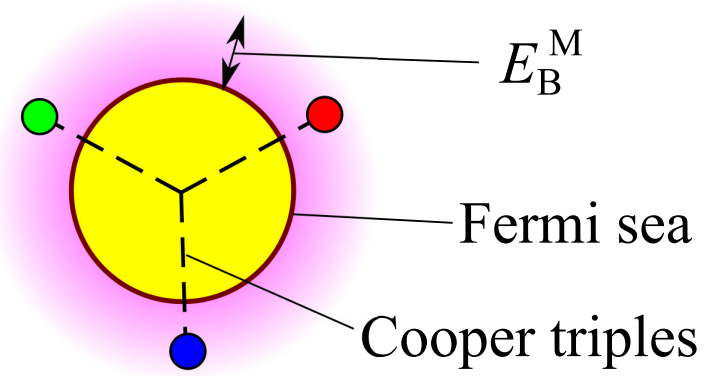

FIG. 1. Schematic figures for the Cooper triple phase in momentum space. The system exhibits a coexistence of the underlying Fermi sea and loosely bound trimers, that is, Cooper triples, near the Fermi surface with the small width of the energy shell typically given by the in-medium trimer binding energy $E_{\mathrm{B}}^{\mathrm{M}}$. Although we work in one dimension, we show higher dimensional configurations for visibility. Note that the Cooper triple phase has been predicted in three dimensions $[41,42]$.

In this work, as a quantum simulator of the hadronquark crossover, we address many-body properties of threecomponent fermions with a three-body attraction. Specifically, we consider the 1D system where a baryonlike three-body bound state is allowed to occur in vacuum even for an infinitesimally small three-body coupling by the scale anomaly. Moreover, a quantum Monte Carlo (QMC) simulation has been performed in 1D recently [40]. As we shall see, the Cooper triple phase occurs at sufficiently large fermion chemical potential, i.e., $\mu \gtrsim E_{\mathrm{B}}$ with the in-vacuum trimer binding energy $E_{\mathrm{B}}$, to ensure the coexistence of the Fermi sea and loosely bound triple states, which is distinct from a simple large-trimer gas conjectured in Ref. [24]. Although mesons have lighter masses $(\simeq 140 \mathrm{MeV})$ than baryonic ones $(\simeq 940$ $\mathrm{MeV}$ ), dense quark (or quarkyonic) matter is dominated by quark and baryonic degrees of freedom due to the Pauli blocking being effective at sufficiently large $\mu_{q}$, leading to a similarity to the present model with the three-body attraction. Moreover, the existence of three-quark attraction has been revealed by the lattice QCD $[55,56]$ and associated Y-shaped color-flux distributions have also been found [57,58].

Short summary-Analyzing three-body spectra, we construct the grand-canonical phase diagram as shown in Fig. 2. We demonstrate that while the Mott temperature $T^{*}$ for the in-medium three-body state is suppressed by thermal agitation [45] around $\mu=0$, it linearly increases with the chemical potential in the high-density regime, indicating the importance of the Fermi surface effect. Such different tendencies between the two regimes lead to the nontrivial crossover from the tightly bound trimer state to Cooper triple phase, which is a three-body counterpart of the BCS to BoseEinstein condensation (BEC) crossover in two-component Fermi gases [59-62]. The Cooper triple phase is characterized by three-body correlations near the atomic Fermi surface, which is analogous to baryonic excitations in quarkyonic matter. Although the present system does not involve gauge fields, the crossover from bound trimers to Cooper triples is reminiscent of the hadron-quark crossover in QCD.

Formalism-We start from a Hamiltonian $H$ for nonrelativistic three-component fermions with a three-body force in

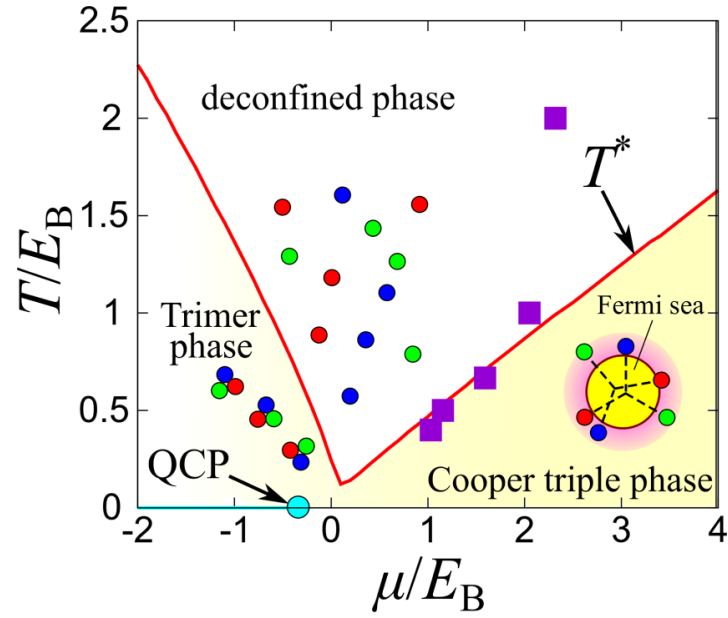

FIG. 2. Grand-canonical phase diagram of one-dimensional three-component fermions with a three-body attraction. $T^{*}$ is the Mott temperature where the in-medium trimer binding energy $E_{\mathrm{B}}^{\mathrm{M}}$ disappears. $\mu=-E_{\mathrm{B}} / 3$ at $T=0$ is a trivial quantum critical point (QCP) for the transition from a zero-density (vacuum) to nonzero-density state. The purple squares show the points where the isothermal compressibility exhibits a minimum as a function of $\mu$ in the QMC results [40].

1D:

$$
\begin{aligned}
H= & \sum_{\gamma=\mathrm{r}, \mathrm{g}, \mathrm{b}} \sum_{p} \xi_{p, \gamma} c_{p, \gamma}^{\dagger} c_{p, \gamma} \\
& +g_{3} \sum_{P, k, q, k^{\prime}, q^{\prime}} c_{\frac{P}{3}+k-\frac{q}{2}, \mathrm{r}}^{\dagger} c_{\frac{P}{3}+q, \mathrm{~g}}^{\dagger} c_{\frac{P}{3}-k-\frac{q}{2}, \mathrm{~b}}^{\dagger} \\
& \times c_{\frac{P}{3}-k^{\prime}-\frac{q^{\prime}}{2}, \mathrm{~b}} c_{\frac{P}{3}+q^{\prime}, \mathrm{g}} c_{\frac{P}{3}+k^{\prime}-\frac{q^{\prime}}{2}, \mathrm{r}},
\end{aligned}
$$

where $\gamma=\mathrm{r}, \mathrm{g}, \mathrm{b}$ denote the internal degrees of freedom of fermions, $\xi_{p, \gamma}=p^{2} /\left(2 m_{\gamma}\right)-\mu_{\gamma}$ is the kinetic energy of a fermion with momentum $p$ and mass $m_{\gamma}$, measured with respect to the chemical potential $\mu_{\gamma}$, and $c_{p, \gamma}^{(\dagger)}$ is the fermionic annihilation (creation) operator. The second term in Eq. (1) denotes the three-body interaction with a contact-type coupling constant $g_{3}$, taken to be negative here. We note that the manipulation of $g_{3}$ has theoretically been proposed in cold atomic [10-13,63] and in Rydberg atomic systems [64,65]. In the Supplemental Material S1 [66] we present one of the possibilities of experimentally realizing this interaction in an atom-trimer resonance model by analogy with the optical Feshbach resonance [67] in connection with a closed-channel trimer state [68-71] and optical control methods [72-76]. For example, applying such a method to an existing mixture (e.g., ${ }^{173} \mathrm{Yb}$ ) with negligibly small two-body interactions away from the Feshbach resonance enables us to obtain a system with the dominant three-body interaction.

Many-body effects are incorporated via the in-medium three-body $T$-matrix $T_{3}^{\mathrm{MB}}\left(P, i \Omega_{n}\right)$, where $P$ is the centerof-mass momentum and $\Omega_{n}=(2 n+1) \pi T$ is the fermion Matsubara frequency with $n \in \mathbb{Z}$. The explicit form of $T_{3}^{\mathrm{MB}}\left(P, i \Omega_{n}\right)$ reads

$$
T_{3}^{\mathrm{MB}}\left(P, i \Omega_{n}\right)=\left[\frac{1}{g_{3}}-\Xi\left(P, i \Omega_{n}\right)\right]^{-1},
$$


where

$$
\Xi\left(P, i \Omega_{n}\right)=\sum_{k, q} \frac{F(k, q, P)}{i \Omega_{n}-\xi_{\frac{P}{3}+k-\frac{q}{2}, \mathrm{r}}-\xi_{\frac{P}{3}+q, \mathrm{~g}}-\xi_{\frac{P}{3}-k-\frac{q}{2}, \mathrm{~b}}} .
$$

The statistical factor $F(k, q, P)$ in Eq. (3) is given by

$$
\begin{aligned}
F(k, q, P)= & \bar{f}_{\frac{P}{3}+k-\frac{q}{2}, \mathrm{r}} \bar{f}_{\frac{P}{3}+q, \mathrm{~g}} \bar{f}_{\frac{P}{3}-k-\frac{q}{2}, \mathrm{~b}} \\
& +f_{\frac{P}{3}+k-\frac{q}{2}, \mathrm{r}} f_{\frac{P}{3}+q, \mathrm{~g}} f_{\frac{P}{3}-k-\frac{q}{2}, \mathrm{~b}},
\end{aligned}
$$

with the Fermi-Dirac distribution function $f_{k, \gamma}=\left(e^{\xi_{k, \gamma} / T}+\right.$ $1)^{-1}$ and $\bar{f}_{k, \gamma}=1-f_{k, \gamma}$. While preceding works allow for the Pauli-blocking effect for the two-body sector [41] only via the first term in Eq. (4) that has the Fermi momentum $k_{\mathrm{F}}$ introduced as a momentum cutoff at $T=0$, the second term, which represents three-hole excitations, is also important at finite temperature [45,77]. By taking $F(k, q, P)=1$, one can reproduce the in-vacuum three-body $T$-matrix $T_{3}\left(P, \Omega_{+}\right)$that appears in a three-body problem. We note that the resummation of specific ladder diagrams for attractive interactions works well even in 1D at finite temperature [78].

We are interested in the conditions that allow a trimer to appear in a medium. The in-vacuum three-body binding energy $E_{\mathrm{B}}=\frac{\Lambda^{2}}{m} e^{\frac{2 \sqrt{3} \pi}{m_{33}}}$ corresponds to the negative energy pole $\Omega=-E_{\mathrm{B}}$ of the in-vacuum three-body $T$-matrix $T_{3}(P=$ $0, \Omega_{+}$) [79] (see also the Supplemental Material S2 [66]), where $\Omega_{+}=\Omega+i \delta$ involves an infinitesimally small imaginary part $i \delta$ with $\delta>0$. Note that binding energy per particle is given by $E_{\mathrm{B}} / 3$. Similarly, the in-medium binding energy $E_{\mathrm{B}}^{\mathrm{M}}$ measured from the bottom of the continuum (i.e., the lowest kinetic energy of three particles at $P=0$ ) can be obtained from

$$
\frac{1}{g_{3}}-\Xi\left(P=0, \Omega=-E_{\mathrm{B}}^{\mathrm{M}}-3 \mu\right)=0 .
$$

Note that a Cooper triple can be defined as a state in which the corresponding pole energy $\Omega+3 \mu=-E_{\mathrm{B}}^{\mathrm{M}}$ is negative at positive $\mu$ and its absolute value is also smaller than $3 \mu$. This is why the regime $E_{\mathrm{B}}^{\mathrm{M}} \ll \mu$ is consistent with the presence of Cooper triples.

Results and discussions-Let us focus hereafter on symmetric three-component fermions $\left(m \equiv m_{\mathrm{r}}=m_{\mathrm{g}}=m_{\mathrm{b}}\right.$ and $\left.\mu \equiv \mu_{\mathrm{r}}=\mu_{\mathrm{g}}=\mu_{\mathrm{b}}\right)$ in one dimension. We determine the Mott temperature $T^{*}[80,81]$ where $E_{\mathrm{B}}^{\mathrm{M}}$ disappears; the result is shown in Fig. 2. Although $T^{*}$ does not imply the presence of any kind of phase transition, it is still worth knowing. Indeed, $T^{*}$ is qualitatively equivalent to the mean-field critical temperature that can be regarded in the context of the BCS-BEC crossover as the temperature where preformed Cooper pairs appear incoherently due to the strong two-body attraction [59-62]. In the numerical calculation we take $E_{\mathrm{B}}^{\mathrm{M}}=$ $10^{-2} E_{\mathrm{B}}$ and $\delta=10^{-3} E_{\mathrm{B}}$ since $\Omega=0$ has a singularity due to the edge of continuum. We confirmed that our estimate of $T^{*}$ is practically unchanged for smaller $\delta$.

Let us turn to in-medium three-body properties at low temperature. In Fig. 3 we display the three-body spectral function $A_{3}(P, \Omega)=-\operatorname{Im} T_{3}^{\mathrm{MB}}\left(P, i \Omega_{n} \rightarrow \Omega_{+}\right)$calculated at $T / E_{\mathrm{B}}=0.1$. Naturally, the medium effect is not significant at low density $[82,83]$. In fact, as depicted in Fig. 3(a) for a typical dilute condition like $\mu / E_{\mathrm{B}}=-1, A_{3}(P, \Omega)$ has a

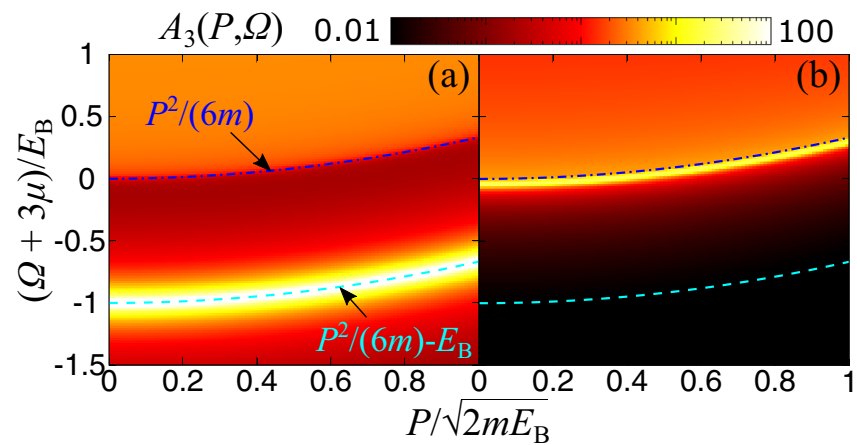

FIG. 3. Three-body spectral functions $A_{3}(P, \Omega)$ calculated at (a) $\mu / E_{\mathrm{B}}=-1$ and (b) $\mu / E_{\mathrm{B}}=2$. The temperature is set at $T / E_{\mathrm{B}}=$ 0.1 in each panel.

strong intensity around the dispersion of a tightly bound trimer given by $\Omega=P^{2} /(6 m)-E_{\mathrm{B}}-3 \mu$, as well as a continuum above $\Omega=P^{2} /(6 m)-3 \mu$. The higher the density, the stronger the medium effect. Consequently, as shown in Fig. 3(b), the bound-state peak in $A_{3}(P, \Omega)$ is strongly suppressed at $\mu / E_{\mathrm{B}}=2$. This suppressed peak, however, does not merge into the continuum at sufficiently low temperature. Instead, the $P=0$ bound-state pole $\Omega=-E_{\mathrm{B}}^{\mathrm{M}}-3 \mu$ remains just below $\Omega=-3 \mu$, which implies the existence of an inmedium trimer near the Fermi surface $\left(0<E_{\mathrm{B}}^{\mathrm{M}} \ll \mu\right)$, that is, a Cooper triple.

We remark that while a molecular state competes with a Cooper triple state in the case of three-component Fermi gases with two-body interactions at finite temperature [45], Cooper triples are not suppressed by such an effect in our model without two-body interactions. Even in the present case, however, Cooper pairs may occur, e.g., due to the effective two-body coupling $g_{2}^{\text {eff }}=g_{3} \rho_{\mathrm{r}}$ between fermions with $\gamma=\mathrm{g}$ and $\mathrm{b}\left(\rho_{\gamma}\right.$ is the number density of $\gamma$ component). This coupling, which may involve a two-body bound state of binding energy $E_{2 \mathrm{~b}}=$ $m\left(g_{2}^{\text {eff }}\right)^{2} / 4$ in $1 \mathrm{D}$, is irrelevant for a large $\Lambda$ since $g_{3}$ and hence $g_{2}^{\text {eff }}$ behave as $\sim 1 / \ln \left(m E_{\mathrm{B}} / \Lambda^{2}\right)$. We remark in passing that in the case of finite-range three-body interactions, irrespective of whether attractive or repulsive [84], $g_{2}^{\text {eff }}$ can be finite and plays a significant role for the interplay between two-body and three-body correlations.

We also note that while a trimer-trimer pairing state used to be invoked as one of the possible ground states in Ref. [24], the trimer-trimer interaction, which was later found to be repulsive [39], would keep Cooper triples unpaired. The repulsive trimer-trimer interaction may lead to the trimer Luttinger liquid (TLL) in the low-density regime at sufficiently low temperature [85]. Our results imply the crossover from the gapless excitation in TLL to the collective mode of Cooper triples with increasing $\mu$. Indeed, a similar crossover of the sound mode has been reported in the 1D BCS-BEC crossover [86]. Finally, we emphasize that our prediction of $T^{*}$ properly allows for thermal agitation, to which the TLL picture is in turn susceptible. From the recent study on finite-temperature Luttinger liquids [87], one may expect the crossover from TLL to the normal trimer or Cooper triple phase with increasing $T$ below $T^{*}$. 


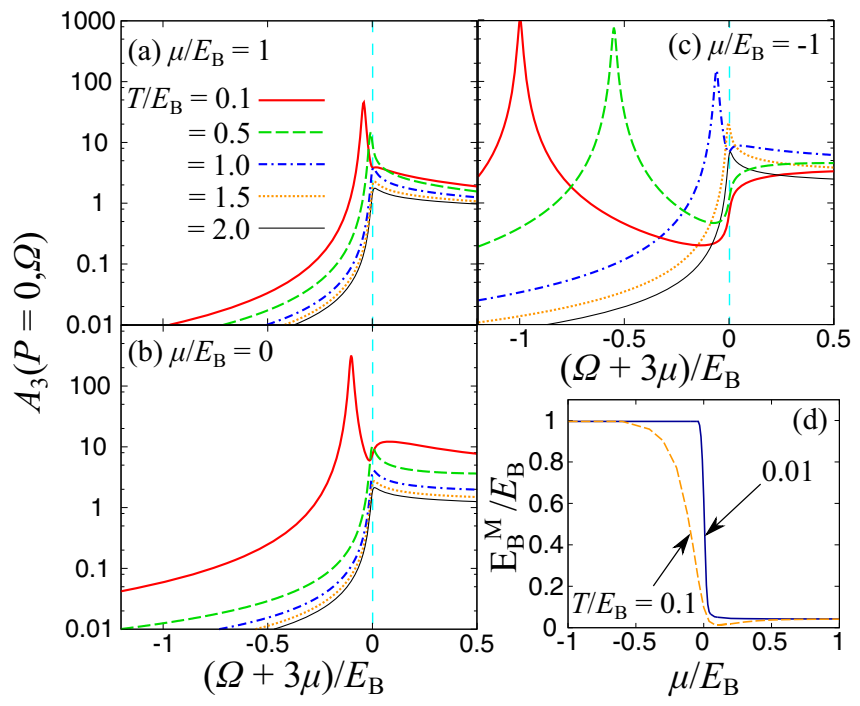

FIG. 4. Temperature dependence of the three-body spectral functions $A_{3}(P=0, \omega)$ at (a) $\mu / E_{\mathrm{B}}=1$, (b) $\mu / E_{\mathrm{B}}=0$, and (c) $\mu / E_{\mathrm{B}}=$ -1 . (d) The in-medium trimer binding energy $E_{\mathrm{B}}^{\mathrm{M}}$ as a function of $\mu$ at $T / E_{\mathrm{B}}=0.01$ and $T / E_{\mathrm{B}}=0.1$.

Figures 4(a)-4(c) show the three-body spectral functions $A_{3}(P=0, \Omega)=-\operatorname{Im} T_{3}^{\mathrm{MB}}\left(P=0, i \Omega_{n} \rightarrow \Omega_{+}\right)$at different temperatures. Even in the case of positive chemical potential $\mu / E_{\mathrm{B}}=1$ depicted in Fig. 4(a), a bound-state peak occurs just below $\Omega+3 \mu=0$ at $T / E_{\mathrm{B}}=0.1$. With increasing temperature, the bound state pole approaches zero energy and eventually merges with the continuum at $T=T^{*}$, which amounts to $\sim 0.5 E_{\mathrm{B}}$ at $\mu / E_{\mathrm{B}}=1$. At $\mu / E_{\mathrm{B}}=0$, as shown in Fig. 4(b), the bound-state peak at $T / E_{\mathrm{B}}=0.1$ is located at a lower energy and also enhanced as compared to the case of positive $\mu$ depicted in Fig. 4(a). At higher temperature, however, the pole at $\mu / E_{\mathrm{B}}=0$ is vulnerable to thermal agitation and hence $T^{*}$ has a minimum around $\mu=0$. At sufficiently low density, $\mu / E_{\mathrm{B}}$ becomes negative. For example, in the case of $\mu / E_{\mathrm{B}}=-1$ depicted in Fig. 4(c), the bound-state pole reduces to $-E_{\mathrm{B}}$ at sufficiently low temperature, a behavior consistent with Fig. 3(a). This is a clear evidence of the presence of tightly bound trimers. When the temperature increases, the bound-state pole approaches zero energy again and finally disappears even in such a low-density regime. Since the system does not form the Fermi surface at negative $\mu$, such a reduction of the trimer binding energy has to be associated with thermal agitation [45].

In Fig. 4(d) we show how $E_{\mathrm{B}}^{\mathrm{M}}$ evolves from the tightly bound trimer phase to the Cooper triple phase at $T / E_{\mathrm{B}}=0.01$ and 0.1. One can see a dramatic drop of $E_{\mathrm{B}}^{\mathrm{M}}$ around $\mu=0$, indicating the change of the system's properties. Both at such low temperatures, $E_{\mathrm{B}}^{\mathrm{M}}$ continuously changes from $E_{\mathrm{B}}$ to the Cooper triple energy $E_{\mathrm{CT}} / E_{\mathrm{B}} \simeq 0.04$ with increasing $\mu$. Here we note that at exactly zero temperature there is a trivial quantum critical point at $\mu / E_{\mathrm{B}}=-1 / 3$ for the transition from a zero-density (vacuum) to nonzero-density state. Note that this critical point is characterized by the effective fugacity $z_{\mathrm{eff}}=$ $e^{\left(3 \mu+E_{\mathrm{B}}\right) / T}$ of a bound trimer [88], as $z_{\mathrm{eff}}$ becomes exactly zero at $T=0$ when $3 \mu<-E_{\mathrm{B}}$. In the absence of $E_{\mathrm{B}}$, this transition would occur at $\mu=0$. From comparison between the results of $T / E_{\mathrm{B}}=0.01$ and $T / E_{\mathrm{B}}=0.1$, one can see that thermal agitation becomes significant around $\mu / E_{\mathrm{B}}=-1 / 3$ to 0 . All these low-temperature properties reflect the fact that while the competition between the three-body binding and the thermal agitation, which is characterized by the ratio $T / E_{\mathrm{B}}$, manifests itself in the low-density regime $(\mu \lesssim 0)$, the formation of Cooper triples in the high-density regime $\left(\mu \gg E_{\mathrm{B}}\right)$ is robust against the thermal agitation due to the Fermi surface effect. The Cooper triple phase in the high-density regime can be identified by a typical energy separation $E_{\mathrm{B}}^{\mathrm{M}} \ll E_{\mathrm{B}} \lesssim \mu$, which is analogous to that in quarkyonic matter. In the lowdensity regime, see the Supplemental Material S3 [66].

We finally revisit the $\mu$ dependence of $T^{*}$ shown in Fig. 2. In the high-density regime, $T^{*}$ linearly increases with increasing $\mu$. Indeed, this behavior is well fitted by the linear function $T^{*}=0.384 \mu+0.095 E_{\mathrm{B}}$. Such a scale-invariant behavior of $T^{*} \propto \mu$ implies that three-body correlations are still alive in the high-density regime. For comparison, in Fig. 2 we plot the points where the QMC result [40] for the isothermal compressibility $\kappa$ normalized by the ideal-gas value $\kappa_{0}$ is minimal with respect to $\mu$. Interestingly, these points coincide well with the $T^{*}-\mu$ relation at low temperature. In QCD, the sound velocity, which is proportional to $\kappa^{-1 / 2}$ at $T=0$, is predicted to be peaked in the hadron-quark crossover regime $[48,49]$. Thus, our results suggest that such macroscopic behavior manifests the emergence of Cooper triples in both systems (for details see the Supplemental Material S4 [66]).

Conclusion-We have clarified the conditions of temperature and chemical potential that allow Cooper triples and trimers to occur in the $1 \mathrm{D}$ equilibrated system of threecomponent fermions with three-body attraction. We have found a nontrivial crossover from the tightly bound trimer phase to the Cooper triple phase with increasing chemical potential, which is analogous to the hadron-quark crossover in QCD. The Mott temperature $T^{*}$ of Cooper triples agrees well with the compressibility minima of the QMC result in this 1D system, implying that the hadron-quark crossover is accompanied by the emergence of quark Cooper triples. Indeed, this scenario is physically analogous to the McLerran-Reddy model for quarkyonic matter [53].

For future perspectives, the comparison of the compressibility between our diagrammatic approach and the existing QMC result would be helpful to confirm the relevance of Cooper triples. Since the deconfined phase near the $T^{*}$ minimum is dominated by strong fluctuations [45], the compressibility anomaly could not be understood by usual quasiparticle pictures. It is also interesting to address quartet condensation [89-92], dual bosonic systems [21,22], higher dimensions [63], effects of two-body interactions [41,42], and lattice systems [93-95]. Moreover, the three-body loss can be a useful probe for the emergence of Cooper triples as in the case of Efimov effects [42,96].

Acknowledgments. We are grateful to Y. Hidaka for reading the manuscript and giving us pertinent comments, and $\mathrm{M}$. Horikoshi, T. Hatsuda, H. Yabu, E. Nakano, J. Takahashi, K. Nishimura, T. Hata, K. Ochi, and S. Akagami for useful discussion. This work is supported by Grants-in-Aid for Scientific Research provided by JSPS through No. 18H01211, No. 18H05406, and No. 20K14480. S.T. was supported by the RIKEN Special Postdoctoral Researchers Program. 
[1] J. Bardeen, L. N. Cooper, and J. R. Schrieffer, Microscopic theory of superconductivity, Phys. Rev. 106, 162 (1957).

[2] H.-W. Hammer, A. Nogga, and A. Schwenk, Colloquium: Three-body forces: From cold atoms to nuclei, Rev. Mod. Phys. 85, 197 (2013).

[3] J. Carlson, V. R. Pandharipande, and R. B. Wiringa, Threenucleon interaction in 3-, 4- and $\infty$-body systems, Nucl. Phys. A 401, 59 (1983).

[4] A. Akmal, V. R. Pandharipande, and D. G. Ravenhall, Equation of state of nucleon matter and neutron star structure, Phys. Rev. C 58, 1804 (1998).

[5] G. Baym, T. Hatsuda, M. Tachibana, and N. Yamamoto, The axial anomaly and the phases of dense QCD, J. Phys. G 35, 104021 (2008).

[6] K. Fukushima, T. Kojo, and W. Weise, Hard-core deconfinement and soft-surface delocalization from nuclear to quark matter, Phys. Rev. D 102, 096017 (2020).

[7] C. Mora, R. Egger, A. O. Gogolin, and A. Komnik, AtomDimer Scattering for Confined Ultracold Fermion Gases, Phys. Rev. Lett. 93, 170403 (2004).

[8] I. E. Mazets, T. Schumm, and J. Schmiedmayer, Breakdown of Integrability in a Quasi-1D Ultracold Bosonic Gas, Phys. Rev. Lett. 100, 210403 (2008).

[9] L. Pricoupenko, Pure confinement-induced trimer in onedimensional atomic waveguides, Phys. Rev. A 97, 061604(R) (2018).

[10] D. S. Petrov, Three-Body Interacting Bosons in Free Space, Phys. Rev. Lett. 112, 103201 (2014).

[11] D. S. Petrov, Elastic multibody interactions on a lattice, Phys. Rev. A 90, 021601(R) (2014).

[12] G. Guijarro, A. Pricoupenko, G. E. Astrakharchik, J. Boronat, and D. S. Petrov, One-dimensional three-boson problem with two- and three-body interactions, Phys. Rev. A 97, 061605(R) (2018).

[13] A. Pricoupenko and D. S. Petrov, Three-body interaction near a narrow two-body zero crossing, Phys. Rev. A 100, 042707 (2019).

[14] Y. Sekino and Y. Nishida, Quantum droplet of one-dimensional bosons with a three-body attraction, Phys. Rev. A 97, 011602(R) (2018).

[15] M. Valiente and V. Pastukhov, Anomalous frequency shifts in a one-dimensional trapped Bose gas, Phys. Rev. A 99, 053607 (2019).

[16] M. Valiente, Three-body repulsive forces among identical bosons in one dimension, Phys. Rev. A 100, 013614 (2019).

[17] N. L. Harshman and A. C. Knapp, Anyons from three-body hard-core interactions in one dimension, Ann. Phys. 412, 168003 (2020).

[18] Y. Nishida, Semisuper Efimov Effect of Two-Dimensional Bosons at a Three-Body Resonance, Phys. Rev. Lett. 118, 230601 (2017).

[19] Y. Horinouchi, Universality of semisuper-Efimov effect, arXiv:2002.07330.

[20] A. Pricoupenko and D. S. Petrov, Higher-order effective interactions for bosons near a two-body zero crossing, Phys. Rev. A 103, 033326 (2021).

[21] M. Valiente, Bose-Fermi dualities for arbitrary one-dimensional quantum systems in the universal low-energy regime, Phys. Rev. A 102, 053304 (2020).
[22] M. Valiente, Universal duality transformations in interacting one-dimensional quantum systems, Phys. Rev. A 103, L021302 (2021).

[23] Y. Sekino and Y. Nishida, Field-theoretical aspects of onedimensional Bose and Fermi gases with contact interactions, Phys. Rev. A 103, 043307 (2021).

[24] J. E. Drut, J. R. McKenney, W. S. Daza, C. L. Lin, and C. R. Ordóñez, Quantum Anomaly and Thermodynamics of OneDimensional Fermions with Three-Body Interactions, Phys. Rev. Lett. 120, 243002 (2018).

[25] O. Bergman, Nonrelativistic field-theoretic scale anomaly, Phys. Rev. D 46, 5474 (1992).

[26] L. P. Pitaevskii and A. Rosch, Breathing modes and hidden symmetry of trapped atoms in two dimensions, Phys. Rev. A 55, R853 (1997).

[27] T. Schäfer and D. Teaney, Nearly perfect fluidity: From cold atomic gases to hot quark gluon plasmas, Rep. Prog. Phys. 72, 126001 (2009).

[28] M. Olshanii, H. Perrin, and V. Lorent, Example of a Quantum Anomaly in the Physics of Ultracold Gases, Phys. Rev. Lett. 105, 095302 (2010).

[29] J. Hofmann, Quantum Anomaly, Universal Relations, and Breathing Mode of a Two-Dimensional Fermi Gas, Phys. Rev. Lett. 108, 185303 (2012).

[30] H. Hu, B. C. Mulkerin, U. Toniolo, L. He, and X.-J. Liu, Reduced Quantum Anomaly in a Quasi-Two-Dimensional Fermi Superfluid: Significance of the Confinement-Induced Effective Range of Interactions, Phys. Rev. Lett. 122, 070401 (2019).

[31] X. Y. Yin, H. Hu, and X.-J. Liu, Few-Body Perspective of a Quantum Anomaly in Two-Dimensional Fermi Gases, Phys. Rev. Lett. 124, 013401 (2020).

[32] E. Vogt, M. Feld, B. Fröhlich, D. Pertot, M. Koschorreck, and M. Köhl, Scale Invariance and Viscosity of a Two-Dimensional Fermi Gas, Phys. Rev. Lett. 108, 070404 (2012).

[33] M. Holten, L. Bayha, A. C. Klein, P. A. Murthy, P. M. Preiss, and S. Jochim, Anomalous Breaking of Scale Invariance in a Two-Dimensional Fermi Gas, Phys. Rev. Lett. 121, 120401 (2018).

[34] T. Peppler, P. Dyke, M. Zamorano, I. Herrera, S. Hoinka, and C. J. Vale, Quantum Anomaly and 2D-3D Crossover in Strongly Interacting Fermi Gases, Phys. Rev. Lett. 121, 120402 (2018).

[35] P. A. Murthy, N. Defenu, L. Bayha, M. Holten, P. M. Preiss, T. Enss, and S. Jochim, Quantum scale anomaly and spatial coherence in a 2D Fermi superfluid, Science 365, 268 (2019).

[36] P. A. Murthy, I. Boettcher, L. Bayha, M. Holzmann, D. Kedar, M. Neidig, M. G. Ries, A. N. Wenz, G. Zürn, and S. Jochim, Observation of the Berezinskii-Kosterlitz-Thouless Phase Transition in an Ultracold Fermi Gas, Phys. Rev. Lett. 115, 010401 (2015).

[37] W. S. Daza, J. E. Drut, C. L. Lin, and C. R. Ordóñez, A quantum field-theoretical perspective on scale anomalies in 1D systems with three-body interactions, Mod. Phys. Lett. A 34, 1950291 (2019).

[38] J. Maki and C. R. Ordóñez, Virial expansion for a threecomponent Fermi gas in one dimension: The quantum anomaly correspondence, Phys. Rev. A 100, 063604 (2019).

[39] J. R. McKenney and J. E. Drut, Fermi-Fermi crossover in the ground state of one-dimensional few-body systems with 
anomalous three-body interactions, Phys. Rev. A 99, 013615 (2019).

[40] J. R. McKenney, A. Jose, and J. E. Drut, Thermodynamics and static response of anomalous one-dimensional fermions via a quantum Monte Carlo approach in the worldline representation, Phys. Rev. A 102, 023313 (2020).

[41] P. Niemann and H.-W. Hammer, Pauli-blocking effects and Cooper triples in three-component Fermi gases, Phys. Rev. A 86, 013628 (2012).

[42] H. Tajima, S. Tsutsui, T. M. Doi, and K. Iida, Three-body crossover from a Cooper triple to a bound trimer state in threecomponent Fermi gases near a triatomic resonance, Phys. Rev. A 104, 053328 (2021).

[43] D. J. MacNeill and F. Zhou, Pauli Blocking Effect on Efimov States near a Feshbach Resonance, Phys. Rev. Lett. 106, 145301 (2011).

[44] N. G. Nygaard and N. T. Zinner, Efimov three-body states on top of a Fermi sea, New J. Phys. 16, 023026 (2014).

[45] H. Tajima and P Naidon, Quantum chromodynamics (QCD)like phase diagram with Efimov trimers and Cooper pairs in resonantly interacting SU(3) Fermi gases, New J. Phys. 21, 073051 (2019).

[46] M. Sun and X. Cui, Efimov physics in the presence of a Fermi sea, Phys. Rev. A 99, 060701(R) (2019).

[47] A. Sanayei and L. Mathey, Three-body bound states of an atom in a Fermi mixture, arXiv:2007.13511.

[48] G. Baym, T. Hatsuda, T. Kojo, P. D. Powell, Y. Song, and T. Takatsuka, From hadrons to quarks in neutron stars: A review, Rep. Prog. Phys. 81, 056902 (2018).

[49] T. Kojo, QCD equations of state and speed of sound in neutron stars, AAPPS Bull. 31, 11 (2021).

[50] L. McLerran and R. D. Pisarski, Phases of dense quarks at large $N_{c}$, Nucl. Phys. A 796, 83 (2007).

[51] Y. Hidaka, L. D. McLerran, and R. D. Pisarski, Baryons and the phase diagram for a large number of colors and flavors, Nucl. Phys. A 808, 117 (2008).

[52] K. Fukushima and T. Kojo, The quarkyonic star, Astrophys. J. 817, 180 (2016).

[53] L. McLerran and S. Reddy, Quarkyonic Matter and Neutron Stars, Phys. Rev. Lett. 122, 122701 (2019).

[54] T. Kojo, Stiffening of matter in quark-hadron continuity, Phys. Rev. D 104, 074005 (2021).

[55] T. T. Takahashi, H. Matsufuru, Y. Nemoto, and H. Suganuma, Three-Quark Potential in SU(3) Lattice QCD, Phys. Rev. Lett. 86, 18 (2001).

[56] T. T. Takahashi, H. Suganuma, Y. Nemoto, and H. Matsufuru, Detailed analysis of the three-quark potential in SU(3) lattice QCD, Phys. Rev. D 65, 114509 (2002).

[57] H. Ichie, V. Bornyakov, T. Streuer, and G. Shierholtz, The flux distribution of the three quark system in SU(3), Nucl. Phys. B 119, 751 (2003).

[58] H. Ichie, V. Bornyakov, T. Streuer, and G. Shierholtz, Flux tubes of two- and three-quark system in full QCD, Nucl. Phys. A 721, C899 (2003).

[59] W. Zwerger (ed.), The BCS-BEC Crossover and the Unitary Fermi Gas, Lecture Notes in Physics Vol. 836 (Springer, Berlin, 2012).

[60] M. Randeria and E. Taylor, Crossover from Bardeen-CooperSchrieffer to Bose-Einstein condensation and the unitary Fermi gas, Annu. Rev. Condens. Matter Phys. 5, 209 (2014).
[61] G. C. Strinati, P. Pieri, G. Röpke, P. Schuck, and M. Urban, The BCS-BEC crossover: From ultra-cold Fermi gases to nuclear systems, Phys. Rep. 738, 3 (2018).

[62] Y. Ohashi, H. Tajima, and P. van Wyk, BCS-BEC crossover in cold atomic and in nuclear systems, Prog. Part. Nucl. Phys. 111, 103739 (2020).

[63] S. Akagami, H. Tajima, and K. Iida, Condensation of Cooper triples, Phys. Rev. A 104, L041302 (2021).

[64] F. M. Gambetta, C. Zhang, M. Hennrich, I. Lesanovsky, and W. Li, Long-Range Multibody Interactions and Three-Body Antiblockade in a Trapped Rydberg Ion Chain, Phys. Rev. Lett. 125, 133602 (2020).

[65] D. P. Ornelas-Huerta, P. Bienias, A. N. Craddock, M. J. Gullans, A. J. Hachtel, M. Kalinowski, M. E. Lyon, A. V. Gorshkov, S. L. Rolston, and J. V. Porto, Tunable Three-Body Loss in a Nonlinear Rydberg Medium, Phys. Rev. Lett. 126, 173401 (2021).

[66] See Supplemental Material at http://link.aps.org/supplemental/ 10.1103/PhysRevResearch.4.L012021 for the possibility of experimentally realizing the three-body interaction, in-vacuum three-body properties, the classical description of a fermiontrimer mixture, and quarkyonic-matter-like model for the ground state.

[67] C. Chin, R. Grimm, P. Julienne, and E. Tiesinga, Feshbach resonances in ultracold gases, Rev. Mod. Phys. 82, 1225 (2010).

[68] M. T. Cvitaš, P. Soldán, J. M. Hutson, P. Honvault, and J.-M. Launay, Interactions and dynamics in $\mathrm{Li}+\mathrm{Li}_{2}$ ultracold collisions, J. Chem. Phys. 127, 074302 (2007).

[69] E. N. Ghassemi, J. Larson, and Å. Larson, A diabatic representation of the two lowest electronic states of $\mathrm{Li}_{3}, \mathrm{~J}$. Chem. Phys. 140, 154304 (2014).

[70] P.-G. Yan, L.-Y. Tang, Z.-C. Yan, and J. F. Babb, Calculations of long-range three-body interactions for $\operatorname{Li}\left(2{ }^{2} S\right)-\operatorname{Li}\left(2{ }^{2} S\right)-\operatorname{Li}(2$ ${ }^{2}$ P), Phys. Rev. A 94, 022705 (2016).

[71] M. Horikoshi (private communication).

[72] H. Wu and J. E. Thomas, Optical Control of Feshbach Resonances in Fermi Gases Using Molecular Dark States, Phys. Rev. Lett. 108, 010401 (2012).

[73] H. Wu and J. E. Thomas, Optical control of the scattering length and effective range for magnetically tunable Feshbach resonances in ultracold gases, Phys. Rev. A 86, 063625 (2012).

[74] A. Jagannathan, N. Arunkumar, J. A. Joseph, and J. E. Thomas, Optical Control of Magnetic Feshbach Resonances by ClosedChannel Electromagnetically Induced Transparency, Phys. Rev. Lett. 116, 075301 (2016).

[75] N. Arunkumar, A. Jagannathan, and J. E. Thomas, Probing Energy-Dependent Feshbach Resonances by Optical Control, Phys. Rev. Lett. 121, 163404 (2018).

[76] N. Arunkumar, A. Jagannathan, and J. E. Thomas, Designer Spatial Control of Interactions in Ultracold Gases, Phys. Rev. Lett. 122, 040405 (2019).

[77] H. Tajima and P. Naidon, Multi-body correlations in SU(3) Fermi gases, J. Low Temp. Phys. 196, 163 (2019).

[78] H. Tajima, S. Tsutsui, and T. M. Doi, Low-dimensional fluctuations and pseudogap in Gaudin-Yang Fermi gases, Phys. Rev. Research 2, 033441 (2020).

[79] When we perform the momentum integration in Eq. (3), we use a transformation $\boldsymbol{q} \rightarrow \frac{2}{\sqrt{3}} \boldsymbol{q}$. Although it modifies the momentum cutoff $\Lambda$ that appears in the expression for $E_{\mathrm{B}}$, by a factor of order unity, $\ln \left(m E_{\mathrm{B}} / \Lambda^{2}\right)$ is not changed up to $O\left(g_{3}^{-1}\right)$. 
[80] P. Schuck, M. Beyer, G. Röpke, W. Schadow, and A. Schnell, Few-body states in Fermi-systems and condensation phenomena, Few Body Syst. Suppl. 12, 50 (2000).

[81] M. Beyer, S. Strauss, P. Schuck, and S. A. Sofianos, Light clusters in nuclear matter of finite temperature, Eur. Phys. J. A 22, 261 (2004).

[82] M. N. Saha, On a physical theory of stellar spectra, Proc. R. Soc. London Sect. A 99, 135 (1921).

[83] K. H. Kingdon and I. Langmuir, The removal of thorium from the surface of a thoriated tungsten filament by positive ion bombardment, Phys. Rev. 22, 148 (1923).

[84] V. Pastukhov, Mean-field properties of impurity in Bose gas with three-body forces, Phys. Lett. A 383, 2610 (2019).

[85] X.-W. Guan, M. T. Batchelor, and C. Lee, Fermi gases in one dimension: From Bethe ansatz to experiments, Rev. Mod. Phys. 85, 1633 (2013).

[86] J. N. Fuchs, A. Recati, and W. Zwerger, Exactly Solvable Model of the BCS-BEC Crossover, Phys. Rev. Lett. 93, 090408 (2004).

[87] F. He, Y.-Z. Jiang, H.-Q. Lin, R. G. Hulet, H. Pu, and X.-W. Guan, Emergence and Disruption of Spin-Charge Separation in One-Dimensional Repulsive Fermions, Phys. Rev. Lett. 125, 190401 (2020).
[88] V. Ngampruetikorn, M. M. Parish, and J. Levinsen, Hightemperature limit of the resonant Fermi gas, Phys. Rev. A 91, 013606 (2015).

[89] H. Kamei and K. Miyake, On quartet superfluidity of fermionic atomic gas, J. Phys. Soc. Jpn. 74, 1911 (2005).

[90] T. Sogo, G. Röpke, and P. Schuck, Many-body approach for quartet condensation in strong coupling, Phys. Rev. C 81, 064310 (2010).

[91] Y. Nishida and D. T. Son, Universal four-component Fermi gas in one dimension, Phys. Rev. A 82, 043606 (2010).

[92] X.-X. Huang, M. Claassen, E. W. Huang, B. Moritz, and T. P. Devereaux, Biexciton Condensation in Electron-Hole-Doped Hubbard Bilayers: A Sign-Problem-Free Quantum Monte Carlo Study, Phys. Rev. Lett. 124, 077601 (2020).

[93] L. Del Re and M. Capone, Selective insulators and anomalous responses in three-component fermionic gases with broken SU(3) symmetry, Phys. Rev. A 98, 063628 (2018).

[94] A. Cristianen and J. Sous, Exploring universal and nonuniversal regimes of trimers from three-body interactions in one-dimensional lattices, Phys. Rev. A 101, 063610 (2020).

[95] L. Gotta, L. Mazza, P. Simon, and G. Roux, Kinetic formation of trimers in a spinless fermionic chain, arXiv:2111.10316.

[96] P. Naidon and S. Endo, Efimov physics: A review, Rep. Prog. Phys. 80, 056001 (2017). 